\section{Photobleaching of YFP does not produce a CFP-like species that affects FRET measurements}

To the editor: Fluorescence resonance energy transfer (FRET) efficiency between variants of fluorescent protein can be determined by photobleaching the acceptor molecule ${ }^{1}$. After complete acceptor bleaching, FRET efficiency equals the dequenched donor fluorescence divided by the total donor fluorescence. Recently, Valentin et al. ${ }^{2}$ reported that photobleaching of yellow fluorescent protein (YFP), Citrine and Venus (commonly used acceptor fluorescent protein tags) produced a species with an emission peak similar to that of cyan fluorescent protein (CFP), a widely-used donor fluorescent protein. This result predicts that FRET efficiency determined by acceptor photobleaching will be overestimated. As this prediction potentially impacts the accuracy of numerous existing studies and raises concerns about the validity of the photobleaching method, we examined the effects of acceptor fluorescent protein photobleaching using several imaging modalities commonly used in FRET experiments.

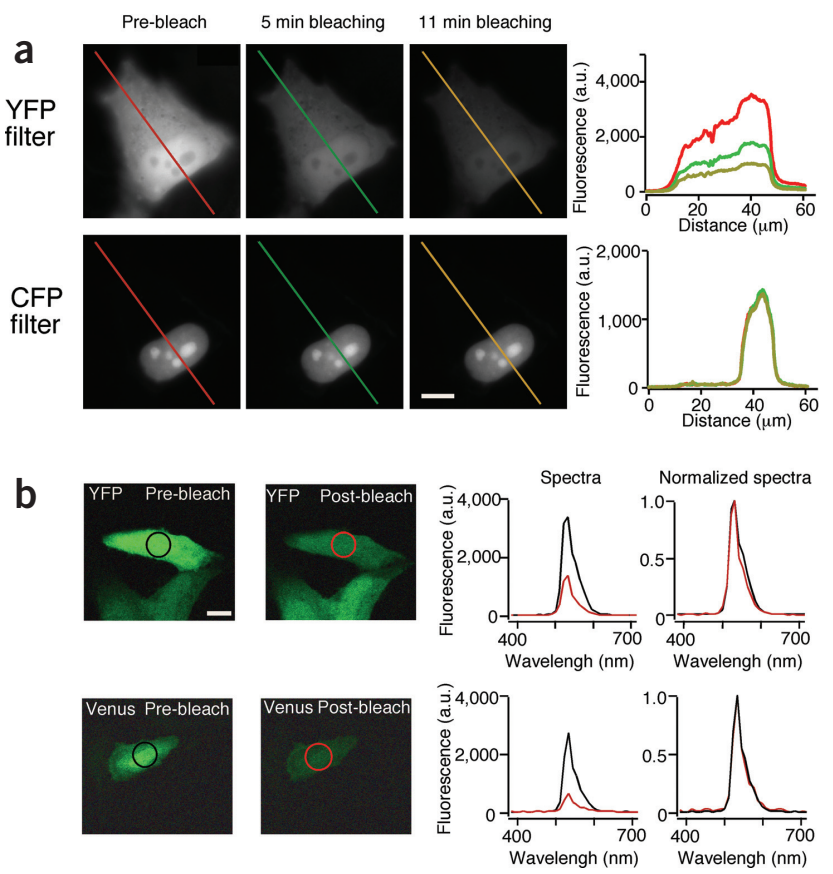

Figure 1 | Photobleaching of Venus and YFP does not produce a CFPlike species. (a) Images were acquired using conventional fluorescence microscopy (60x oil objective, numerical aperture (NA) 1.4). The lines in the images represent where the fluorescence intensity profiles were measured. (b) The emission spectral images were acquired using two-photon excitation at $940 \mathrm{~nm}$ as described in the text. Detailed procedures are described in Supplementary Methods. Scale bar, $10 \mu \mathrm{m}$.
We imaged HeLa cells coexpressing Venus and nuclear-localized CFP one day after transfection (Fig. 1a; Supplementary Methods online). Prolonged illumination through a YFP filter set (excitation: $500 \pm 10 \mathrm{~nm}$, emitter: $540 \pm 15 \mathrm{~nm}$, dichroic: $520 \mathrm{LP}$ ) decreased the intensity of Venus fluorescence (YFP filter set) evenly throughout the cell. Conversely, the pattern and intensity of fluorescence detected through a CFP filter (excitation: $436 \pm 10$ nm, emitter: $480 \pm 20 \mathrm{~nm}$, dichroic: 455LP) remained unchanged. Additionally, fixation of cells expressing YFP or Venus with paraformaldehyde followed by photobleaching (50\% decrease in YFP channel intensity) did not produce an increase in CFP channel fluorescence intensity (Supplementary Fig. 1 online). We obtained similar results (Supplementary Fig. 1) using CFP excitation (excitation: $406 \pm 7.5 \mathrm{~nm}$ ) similar to that used by Valentin et al. ${ }^{2}$.

We determined the emission spectra of either Venus or YFP before and after photobleaching ( $514 \mathrm{~nm}$ argon ion laser) of fixed HeLa cells expressing these proteins. Photobleaching of Venus or YFP $(>50 \%)$ did not alter the shape of the emission spectra acquired with an LSM510 confocal microscope using two-photon excitation between 750 and $940 \mathrm{~nm}$ (Fig. 1b, and Supplementary Figs. 1 and 2 online). In particular, we observed no detectable increase in fluorescence intensity from $420-500 \mathrm{~nm}$. This finding is in marked contrast to the result of Valentin et al. ${ }^{2}$.

Our results demonstrate that photobleaching of YFP or Venus, under the conditions stated, does not produce CFP-like fluorescence, and thus FRET efficiency determination using acceptor photobleaching need not be encumbered by the reported phenomenon ${ }^{2}$. It is unclear why our results deviate from those of Valentin et al. ${ }^{2}$ as experimental conditions were similar (but not identical).

Note: Supplementary information is available on the Nature Methods website.

\section{Christopher Thaler ${ }^{1}$, Steven S Vogel ${ }^{1}$, Stephen R Ikeda ${ }^{2}$ \& Huanmian Chen ${ }^{2}$ \\ Laboratory of Molecular Physiology, Sections on ${ }^{1}$ Cellular Biophotonics and \\ ${ }^{2}$ Transmitter Signaling, National Institute on Alcohol Abuse and Alcoholism, \\ National Institutes of Health, Bethesda, Maryland 20892, USA. e-mail: huanchen@mail.nih.gov \\ 1. Kenworthy, A. K. Methods 24, 289-296 (2001). \\ 2. Valentin, G. et al. Nature Methods 2, 801 (2005).}

To the editor: Fluorescence resonance energy transfer (FRET) between cyan and yellow fluorescent protein (CFP and YFP) fusion proteins assessed as sensitized emission can be effectively controlled by measuring dequenching of CFP fluorescence after YFP photobleaching. Recently Valentin et al. ${ }^{1}$ reported that photobleaching of YFP induced the formation of a fluorescent product excitable at $405 \mathrm{~nm}$ with an emission maximum similar to that of CFP. This could severely affect measurements of FRET between CFP and YFP fusion proteins based on donor dequenching after acceptor photobleaching. Therefore, we have tested whether photoconversion of YFP interferes with CFP-dequenching during 
a

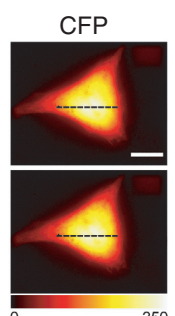

$\stackrel{0}{0} \begin{aligned} & 350 \\ & \text { Fluorescence }(\text { a.u. }\end{aligned}$
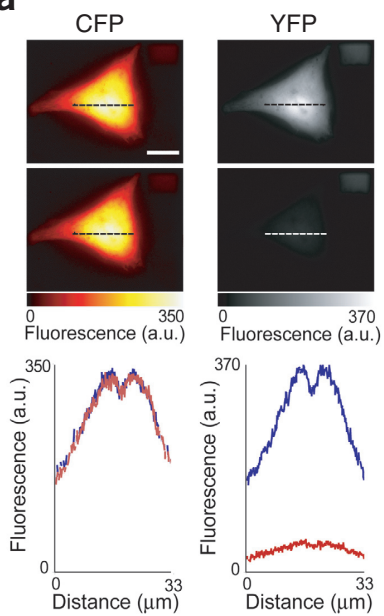

C
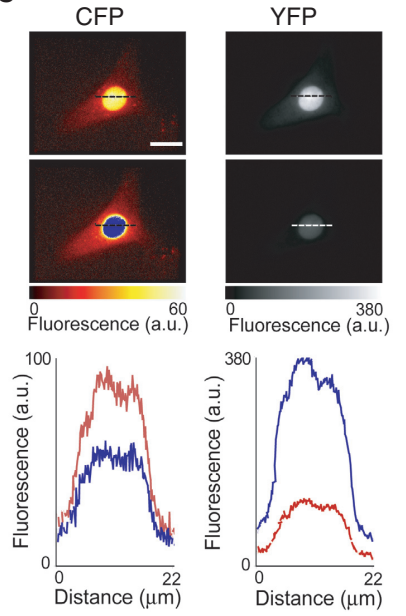

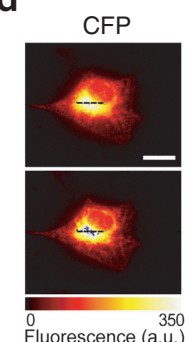

b
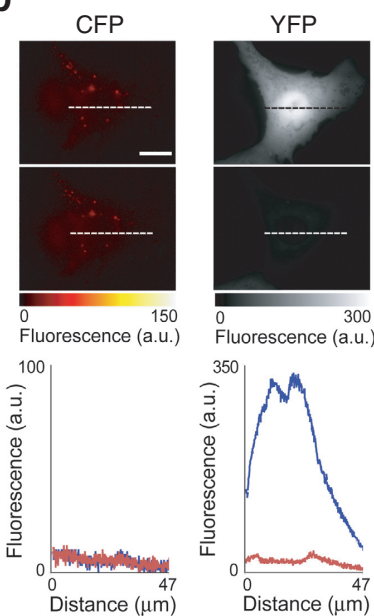

d

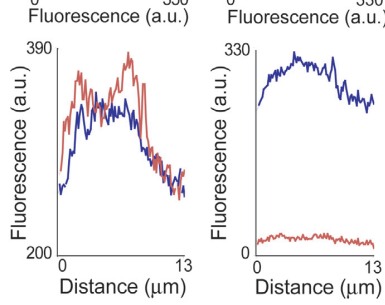

Figure 1 | Photoconversion of YFP into CFP-like species is not detectable under standard conditions. (a-d) Photobleaching of YFP in live Vero cells coexpressing unfused CFP and YFP (a) or only YFP (b) does not generate measurable 'CFP-like' fluorescence, whereas it does in cells showing intramolecular FRET (c) or interaction between a pair of CFP and YFP fusion proteins (d). YFP fluorescence (right) or CFP fluorescence (left) before (top) and after (bottom) photobleaching. Scale bars, $20 \mu \mathrm{m}$. Fluorescence intensities along the line scans (dashed lines in images) are shown before (blue lines) and after (red lines) YFP photobleaching. YFP photobleaching was performed at $532 \mathrm{~nm}$; for details see Supplementary Methods.

photobleaching of YFP at $532 \mathrm{~nm}$ in live cells (Supplementary Methods online). Coexpression of non-fused CFP and YFP in Vero cells gave negligible sensitized emission. Correspondingly, photobleaching of YFP did not result in a measurable increase in CFP fluorescence (Fig. 1a). Cells expressing only YFP also did not show 'CFP-like' fluorescence upon YFP photobleaching (Fig. 1b). In contrast, cells expressing a protein in which CFP and YFP were in the same molecule, separated only by 15 amino acids, exhibited a strong donor dequenching effect (Fig. 1c). Coexpression of two interacting SNARE-CFP and -YFP fusion proteins also resulted in rather weak but substantial donor dequenching upon YFP-photobleaching (Fig. 1d). As the reported data ${ }^{1}$ had been obtained with fixed cells, we performed

similar experiments in fixed Vero cells, but photobleaching of YFP again did not result in increased 'CFP-like' fluorescence (Supplementary Fig. 1 online). Photobleaching of YFP-expressing live or fixed cells at 514 instead of $532 \mathrm{~nm}$ again did not increase CFP-like fluorescence (Supplementary Fig. 2 online). Finally, spectrofluorimetry with live cells expressing either Venus $^{2}$ alone or coexpressing Venus and GFP2 fusion proteins, showed also that photobleaching of Venus at $530 \mathrm{~nm}$ did not generate a measurable CFP-like product (Supplementary Fig. 3 online). In case of interacting Venus and GFP2 fusion proteins Venus photobleaching was associated with a clear increase of GFP2 fluorescence indicating FRET but without a fluorescence increase in the spectral range of CFP. McAnaney et al. ${ }^{3}$ reported that at $\mathrm{pH} 7.2$ irradiation of YFP at $514 \mathrm{~nm}$ for $10 \mathrm{~min}$ led to about $80 \%$ photobleaching, which was accompanied by a small increase in fluorescence between 430 and $500 \mathrm{~nm}$ during excitation at $390 \mathrm{~nm}$, which in contrast to the data of Valentin et al. ${ }^{1}$ did not show a distinct maximum at about $470 \mathrm{~nm}$.

Furthermore, it appears unlikely that the described discrepancies are due to the use of different cell types, as we also did not observe the photoconversion phenomenon described by Valentin et al. ${ }^{1}$ in COS7, NIH3T3 and HEK293 cells. In summary, we can exclude that under standard conditions, photoconversion of YFP (or YFP-related fluorescent proteins) into a compound with CFP-like fluorescent properties interferes with FRET measurement, between pairs of CFP and YFP fusion proteins, by donor dequenching.

Note: Supplementary information is available on the Nature Methods website.

\section{Sophie E Verrier ${ }^{1}$ \& Hans-Dieter Söling ${ }^{1,2}$}

${ }^{1}$ Max Planck Institute of Biophysical Chemistry, Department of Neurobiology,

Am Fassberg 11, D-37077 Göttingen, Germany. ${ }^{2}$ Deceased.

e-mail: sophie.verrier@curie.fr

1. Valentin, G. et al. Nat. Methods 2, 801 (2005).

2. Nagai, T. et al. Nat. Biotechnol. 20, 87-90 (2002).

3. McAnaney, T. B. et al. Biochemistry 44, 5510-5524 (2005).

Valentin and colleagues respond: Verrier \& Söling ${ }^{1}$ and Thaler et $a .^{2}$ did not observe yellow fluorescent protein (YFP) to cyan fluorescent protein (CFP) photoconversion during YFP photobleaching, questioning our previous results ${ }^{3}$. To exclude the possibility that photoconversion was specific to our experiments, we tested different microscopes, constructs and cells, including the sample used in Supplementary Figure $2 \mathbf{b}$ of the Verrier \& Söling report. We detected photoconversion in all cases (Supplementary Fig. 1 online), indicating that it occurred independently of the cell lines, cell preparation and microscope used.

Photoconversion is dependent on the bleaching conditions. Thus, in many experiments of the Verrier \& Söling (Fig. 1, Supplementary Figs. 1 and 3) and Thaler et al. (Fig. 1a and Supplementary Fig. 1a) reports, the bleaching power was probably unsufficient to produce detectable levels of photoconversion (bleaching time between 1 and $10 \mathrm{~min}$, as opposed to tens of seconds with a confocal).

To analyze the impact of photoconversion on fluorescence resonance energy transfer (FRET) measurements, we compared YFP with a CFP-YFP tandem construct, which shows strong FRET. Using cells expressing similar levels of YFP, we observed the following (Supplementary Table 1 online): when CFP was excited at $405 \mathrm{~nm}$, photoconversion accounted for almost 50\% (medium 